Article

\title{
Investigation on Super-Resolution Focusing Performance of a TE-Polarized Nanoslit-Based Two-Dimensional Lens
}

\author{
Yechuan Zhu ${ }^{1, *}$, Shun Zhou ${ }^{1}$, Zhiheng Wang ${ }^{1}$, Yiting Yu ${ }^{2, *}$, Weizheng Yuan ${ }^{2}$ and Weiguo Liu ${ }^{1}$ \\ 1 Shaanxi Province Key Laboratory of Thin Films Technology and Optical Test, Xi'an Technological University, \\ Xi'an 710021, China; zsemail@126.com (S.Z.); wzh_chibakyousuke@163.com (Z.W.); wgliu@163.com (W.L.) \\ 2 Key Laboratory of Micro/Nano Systems for Aerospace, Ministry of Education, Northwestern Polytechnical \\ University, Xi'an 710072, China; yuanwz@nwpu.edu.cn \\ * Correspondence: zyc_xatu@126.com (Y.Z.); yyt@nwpu.edu.cn (Y.Y.)
}

Received: 23 November 2019; Accepted: 16 December 2019; Published: 18 December 2019

check for updates

\begin{abstract}
Conventional optics suffer from the diffraction limit. Our recent work has predicted a nanoslit-based two-dimensional (2D) lens with transverse-electric (TE) polarized design that is capable of realizing the super-resolution focusing of light beyond the diffraction limit in the quasi-far field. Furthermore, the super-resolution capability can be kept in a high-refractive-index dielectric over a wide wavelength range from ultraviolet to visible light. Here, we systematically investigate the influence of various factors on the super-resolution focusing performance of the lens. Factors such as lens aperture, focal length and nanoslit length are considered. In particular, the influence of nanoslit length on lens focusing was ignored in the previous reports about nanoslit-based 2D lenses, since nanoslit length was assumed to be infinite. The numerical results using the finite-difference time-domain (FDTD) method demonstrate that the super-resolution focusing capability of a nanoslit-based 2D lens increases with the lens aperture and reduces with the increase of the lens focal length. On the other hand, it is notable that the length of the lens focus is not equal to but smaller than that of the nanoslits. Therefore, in order to achieve a desired focus length, a lens should be designed with longer nanoslits.
\end{abstract}

Keywords: super-resolution focusing; nanoslit-based 2D lens; influencing factors; focus length

\section{Introduction}

The spatial resolution of conventional optical devices is restricted by diffraction to nearly half the operating wavelength, which greatly limits the performance of all the imaging and focusing systems that lie at the heart of modern biology, electronics and optical integrated circuits [1-3]. For more than a century, many efforts have been made to overcome the diffraction limit. In recent years, the interaction between matter such as nanostructures and light has been extensively studied by various research groups, offering possibilities for solving the problem. In 2005, taking advantage of the enhancement of evanescent components of an illuminated object via the excitation of surface plasmon polaritons (SPPs) [4], a superlens built with an ultrathin silver film was developed to achieve imaging that breaks the diffraction limit with a resolution of $\lambda / 6$ ( $\lambda$ is the operation wavelength) [5]. Moreover, super-resolution focusing was also realized based on metal and dielectric nanostructures [6-10]. These imaging and focusing devices with the capability of overcoming the diffraction limit open great opportunities for the development of a new class of nano-optical devices and techniques. However, the working distance of these devices is greatly confined to the near-field region, or the focus locates inside the structure, which limits the feasibility for actual application. 
In order to achieve optical focusing at a greater distance, a large number of planar lenses formed by metallic and dielectric nanostructures with nanostructures used to modulate light have been proposed, such as metallic nanoslits [11-17], metallic cross-shaped apertures [18], metallic antennas [19], dielectric waveguides [20] and dielectric pillars [21-27]. Few of these planar lenses could overcome the diffraction limit to realize super-resolution focusing. In 2017, by exciting SPPs and enabling them to couple with radiating propagation modes, a conical two-dimensional (2D) plasmonic-zone plate lens was proposed to realize far-field super-resolution focusing [28]. In our latest research, without the excitation of SPPs, we successfully designed a planar lens composed of metallic nanoslits under the illumination of a TE-polarized plane wave (the polarization parallel to the nanoslit) with a capability of super-resolution optical focusing over a larger distance than twice the operation wavelength [29]. In this article, we extensively explore the influence of various factors on the super-resolution focusing performance of the TE-polarized nanoslit-based 2D lens in order to further improve the method and provide a guide for practical applications.

\section{Design and Super-Resolution Focusing of a TE-Polarized Nanoslit-Based 2D Lens}

The lens we consider here comprises an array of nanoslits perforated in a gold film on a glass substrate with TE-polarized design [29], which operates in cedar oil under the normal incidence of a plane wave at the wavelength of $405 \mathrm{~nm}$ from the glass substrate along the $x$ direction, as illustrated in Figure 1. At this wavelength, the relative permittivities of gold and cedar oil are $-1.6745+5.7286 i$ [30] and $2.2952(n=1.5150)$, respectively. All the nanoslits have the same length $l ; t$ is the thickness of the gold film, and $f$ is the desired focal length.

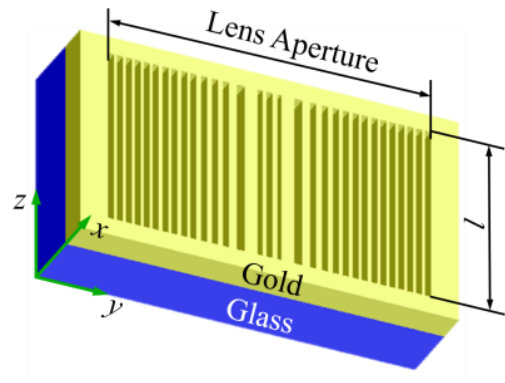

(a)

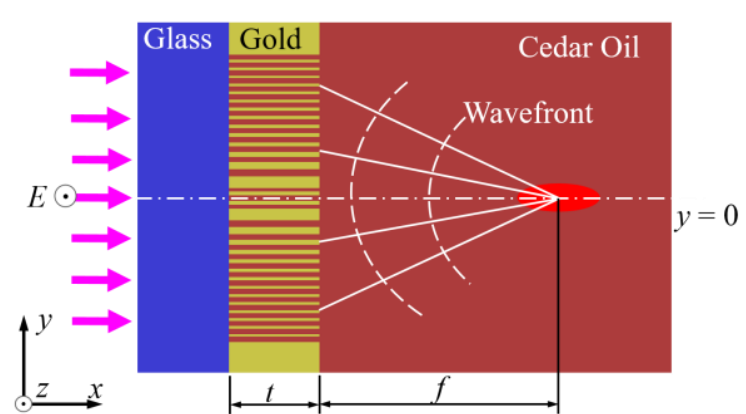

(b)

Figure 1. Structure and focusing scheme of a transverse-electric (TE) polarized nanoslit-based two-dimensional (2D) lens. (a) The structure of the lens is formed by an array of nanoslits perforated in a gold film located on a glass substrate. (b) Schematic focusing of the lens; $t$ is the thickness of metal film.

In our design, the lens structure is symmetric with respect to central nanoslit at $y=0$. The basic building unit is a nanoslit surrounded by gold walls, as shown in the inset of Figure $2 \mathrm{a}$. Here, $w$ is the width of the nanoslit. The thickness of gold film $t=200 \mathrm{~nm}$. 
(a)

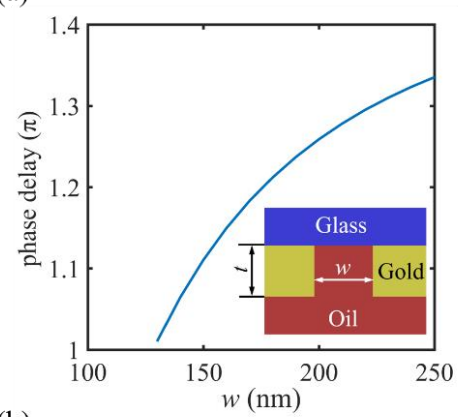

(b)

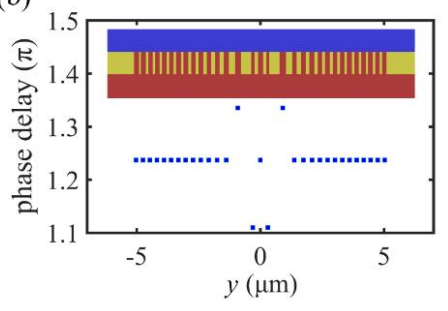

(c)

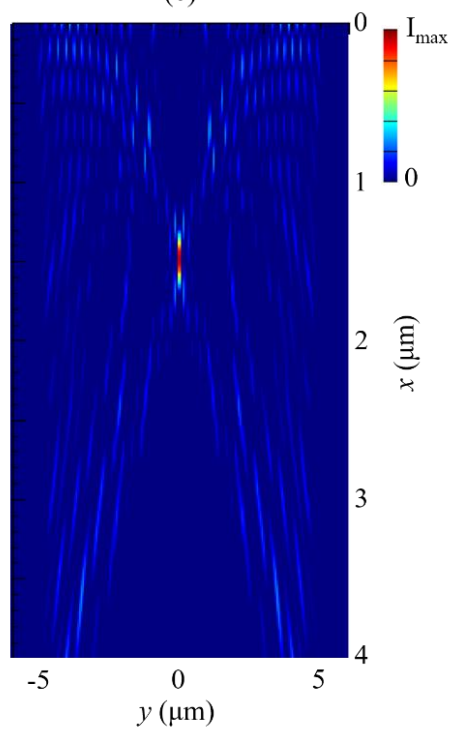

Figure 2. Design and optical focusing of a TE-polarized nanoslit-based 2D lens. (a) Phase delay of a 200-nm-deep nanoslit as a function of nanoslit width $w$ for the operating wavelength of $405 \mathrm{~nm}$. (b) Geometry of the lens (inset) and the phase delay of each nanoslit calculated based on Equation (2). (c) Finite-difference time-domain (FDTD) simulated electric field intensity $|E|^{2}$ of the lens. The plane $x$ $=0$ is the exit surface of the lens, and nanoslit length $l$ is assumed to be infinite.

When a TE-polarized plane wave is normally incident on a metallic nanoslit, multiple TE modes can be transmitted through the nanoslit. In order to obtain desired focusing performance, the zeroth mode $\mathrm{TE}_{0}$ is considered for the lens design. Therefore, the width of all the nanoslits should be above the cutoff width for $\mathrm{TE}_{0}$ mode but below that for the $\mathrm{TE}_{1}$ mode, which should meet the following condition:

$$
\frac{2 \arctan \sqrt{-\varepsilon_{m} / \varepsilon_{d}}}{k \sqrt{\varepsilon_{d}}}<w<\frac{\pi+2 \arctan \sqrt{-\varepsilon_{m} / \varepsilon_{d}}}{k \sqrt{\varepsilon_{d}}},
$$

where $k$ is the free-space wavevector and $\varepsilon_{m}$ and $\varepsilon_{d}$ are the relative permittivities of gold and cedar oil, respectively. The $\mathrm{TE}_{0}$ mode of a nanoslit follows the dispersion relationship:

$$
\frac{w}{2} \sqrt{\varepsilon_{d} k^{2}-\beta_{0}^{2}}=\arctan \frac{\sqrt{\beta_{0}^{2}-\varepsilon_{m} k^{2}}}{\sqrt{\varepsilon_{d} k^{2}-\beta_{0}^{2}}}
$$

where $\beta_{0}$ is the propagation constant for the $\mathrm{TE}_{0}$ mode. The above equation links $\beta_{0}$ to the free-space propagation constant $k$, the nanoslit width $w$, and the permittivities $\varepsilon_{m}$ and $\varepsilon_{d}$. Thus, the phase delay introduced by a nanoslit can be predicted by $\operatorname{Re}\left(\beta_{0}\right) t$. Figure 2a illustrates the phase delay of a nanoslit as a function of nanoslit width. It can clearly be seen that the phase delay strongly depends on nanoslit width. Therefore, we can modulate nanoslit width to control phase delay.

On the other hand, according to the principle of optical interference, the focusing of light at the desired focal distance $f$ from a lens can be realized, provided that the phase delay of each nanoslit matches the required phase difference $(\Delta \varphi(y)-\Delta \varphi(0))$ as a function of the position $y$, which is calculated by:

$$
\Delta \varphi(y)-\Delta \varphi(0)=2 m \pi+\frac{2 \pi n_{d} f}{\lambda}-\frac{2 \pi n_{d} \sqrt{f^{2}+y^{2}}}{\lambda},
$$

where $\lambda$ is the free-space wavelength, $m$ is an arbitrary integer and $n_{d}$ is the refractive index of the working dielectric for the lens.

Based on Equations (2) and (3), the lens immersed in cedar oil with the desired focal length of $1.5 \mu \mathrm{m}$ was designed, as shown in the inset of Figure $2 \mathrm{~b}$. The geometry of the lens is symmetric with 
respect to the plane $y=0$. Beginning from $y=0$ (the lens center), the position sequence of nanoslits is: 0, 0.304, 0.910, 1.376, 1.746, 2.086, 2.408, 2.718, 3.020, 3.316, 3.606, 3.894, 4.180, 4.462, 4.744 and $5.024 \mu \mathrm{m}$. The first three nanoslits have widths of 190, 130 and $250 \mathrm{~nm}$, and all the others are $190 \mathrm{~nm}$. The phase delay of each nanoslit is given in Figure $2 \mathrm{~b}$. Nanoslit length $l$ is assumed to be infinite.

In order to characterize the focusing performance of the designed lens, full electromagnetic field simulations were performed using a commercial finite-difference time-domain (FDTD) solver (Lumerical Solutions, Inc., Vancouver, BC, Canada). In the simulations, the unit length of FDTD cells was set to $2 \mathrm{~nm}$ in both $x$ and $y$ directions to ensure the convergence of the computation and to model the fine features of the electromagnetic field. Perfectly matched layers as the absorbing boundary conditions were employed around the computational domain. The incident TE-polarized plane wave was defined by setting the electric field component of $E_{z}$ with the amplitude equaling one.

The simulated electric field intensity pattern of the lens is shown in Figure 2c, which obviously verifies optical focusing behavior. The realized focal length is $1.484 \mu \mathrm{m}(3.664 \lambda)$, demonstrating excellent agreement with the design value. The full width at half maximum (FWHM) of the focus is $98 \mathrm{~nm}$, about $\lambda / 4.13$, well below the diffraction limit $\left(\lambda /\left(2 n_{d}\right)=\lambda / 3.03=134 \mathrm{~nm}\right)$. Therefore, the super-resolution focusing of light in the quasi-far field is achieved. Moreover, the length of the line focus is identical with the nanoslit length, on account that it is assumed to be infinite.

\section{Effect of Various Factors on the Nanoslit-Based 2D Lens Super-Resolution Focusing}

We firstly explore the effect of nanoslit length $l$ on the focusing properties of the nanoslit-based $2 \mathrm{D}$ lens, since the actual nanoslit length cannot be infinite. Figure 3 gives the focusing properties of the TE-polarized nanoslit-based lens with nanoslit length $l=4 \mu \mathrm{m}$. It can be clearly seen that the length of line focus is approximately $3 \mu \mathrm{m}$, namely 75\% of the nanoslit length (as shown in Figure 3a). Meanwhile, the maximum field strength is not at the center of the focal plane but near the sides (as shown in Figure 3b). Moreover, the FWHMs of line focus can be kept nearly invariable at different positions, as illustrated in Figure 3c,d.

Moreover, the length of the line focus approaches the nanoslit length when the nanoslit length becomes larger. Figure 4 shows the focusing properties of the TE-polarized nanoslit-based 2D lens with nanoslit length $l=15 \mu \mathrm{m}$. The length of the line focus is about $14 \mu \mathrm{m}$, namely, $93.33 \%$ of the nanoslit length. Because the length of line focus is smaller than the nanoslit length, the real value of the focus length of a nanoslit-based 2D lens should be considered in actual application. 

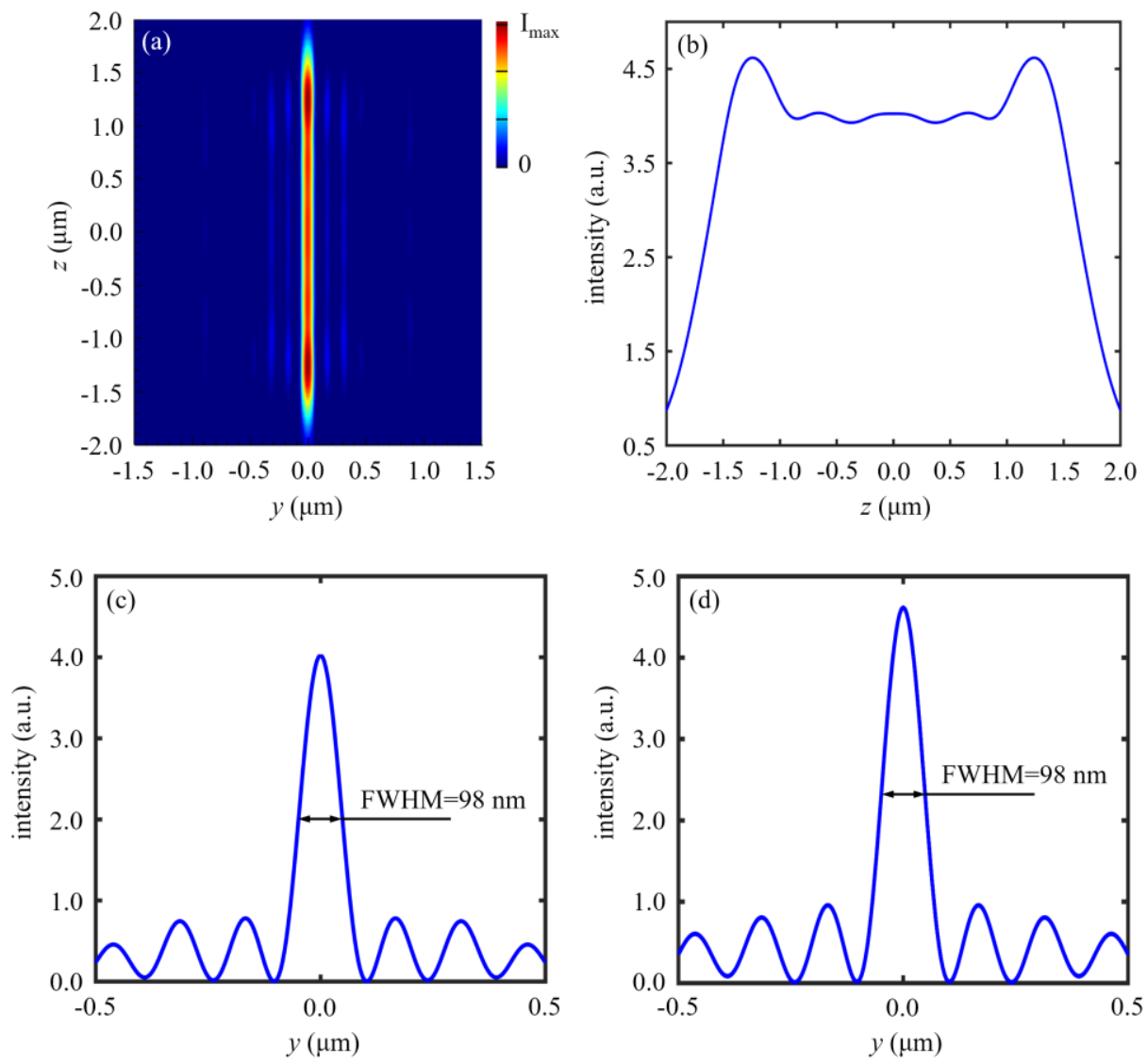

Figure 3. Focusing properties of the TE-polarized nanoslit-based 2D lens with nanoslit length $l=4 \mu \mathrm{m}$. Simulated electric field intensity $|E|^{2}$ distributed (a) at the focal plane and (b) on the line $y=0$ in Figure 3a. (c) The full width at half maximum (FWHM) of the focus located at the lens center $(z=0)$. (d) The FWHM of the focus located at the position of maximum field strength.
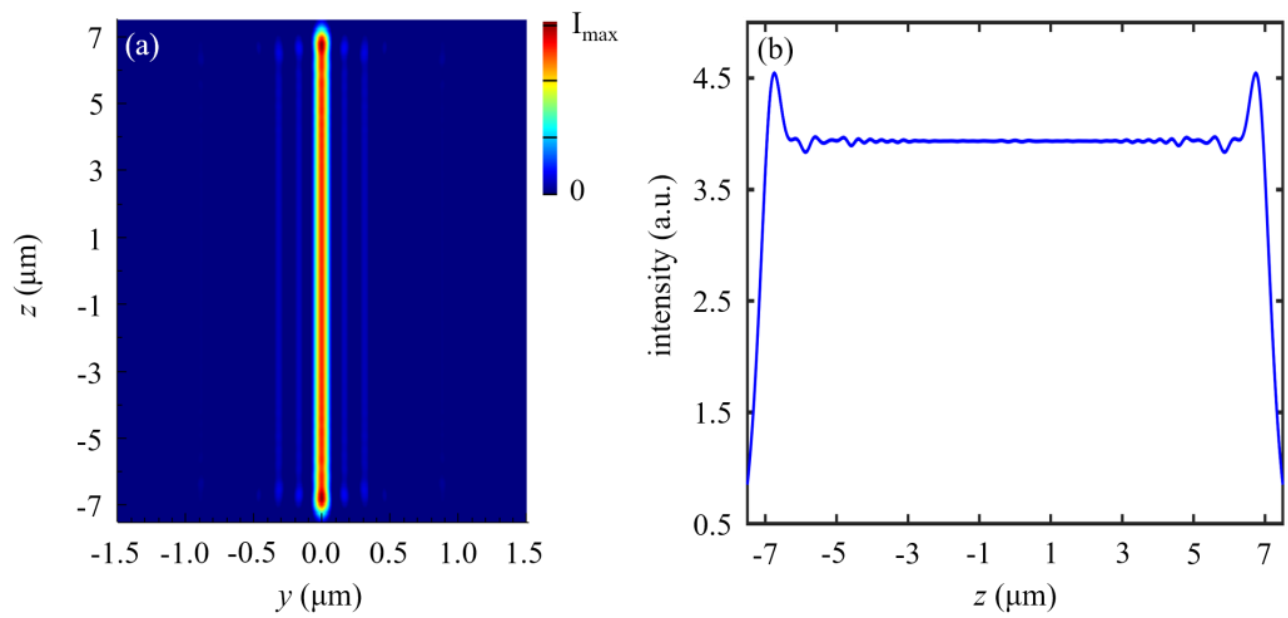

Figure 4. Focusing properties of the TE-polarized nanoslit-based 2D lens with nanoslit length $l=15 \mu \mathrm{m}$. Simulated electric field intensity $|E|^{2}$ distributed (a) at the focal plane and (b) on the line $y=0$ in Figure 4a.

From the above analysis, we can see that the nanoslit length has a significant impact on the focus length of a nanoslit-based 2D lens. On the other hand, the focal size (namely, FWHM) that judges super-resolution capability depends on the aperture and focal length of the lens. Utilizing the same 
design procedure used in Section 2, a series of nanoslit-based 2D lenses with different apertures was built, which also operate in cedar oil with the designed focal length of $1.5 \mu \mathrm{m}$.

Table 1 illustrates the simulated focusing performances of these lenses. For the lens with a small aperture of $2.942 \mu \mathrm{m}$, the real focal length is $1.444 \mu \mathrm{m}$. The deviation from the design value is $3.73 \%$, and the FWHM is $160 \mathrm{~nm}(\lambda / 2.53)$, larger than the diffraction limit of $134 \mathrm{~nm}(\lambda / 3.03)$. Therefore, for this case, the lens cannot break the diffraction limit. When the lens aperture increases, the super-resolution focusing capability of a lens can be improved and the focal length is also close to the design value, as shown in Table 1. From Figure 5a, it can be clearly observed that the FWHM rapidly decreases and gradually flattens out when the lens aperture increases from $2.942 \mu \mathrm{m}$ to $13.550 \mu \mathrm{m}$.

Table 1. The derived focusing performance of nanoslit-based 2D lenses with different lens sizes.

\begin{tabular}{ccccc}
\hline $\begin{array}{c}\text { Lens Aperture } \\
(\mu \mathrm{m})\end{array}$ & $\begin{array}{c}\text { Designed Focal } \\
\text { Length }(\boldsymbol{\mu m})\end{array}$ & $\begin{array}{c}\text { Simulated Focal } \\
\text { Length }(\boldsymbol{\mu m})\end{array}$ & FWHM (nm) & $\begin{array}{c}\text { Max. Intensity } \\
(\mathbf{a} . \mathbf{u} .)\end{array}$ \\
\hline 2.942 & & 1.444 & $160(\lambda / 2.53)$ & 0.918 \\
4.362 & & 1.472 & $126(\lambda / 3.21)$ & 1.719 \\
5.626 & & 1.480 & $114(\lambda / 3.55)$ & 2.264 \\
6.822 & 1.488 & $104(\lambda / 3.89)$ & 2.680 \\
7.978 & 1.5 & 1.488 & $102(\lambda / 3.97)$ & 3.146 \\
9.114 & & 1.484 & $100(\lambda / 4.05)$ & 3.677 \\
10.238 & & 1.484 & $98(\lambda / 4.13)$ & 4.025 \\
11.350 & & 1.484 & $96(\lambda / 4.22)$ & 4.280 \\
12.454 & 1.484 & $95(\lambda / 4.26)$ & 4.607 \\
13.550 & & 1.484 & $94(\lambda / 4.31)$ & 4.921 \\
\hline
\end{tabular}

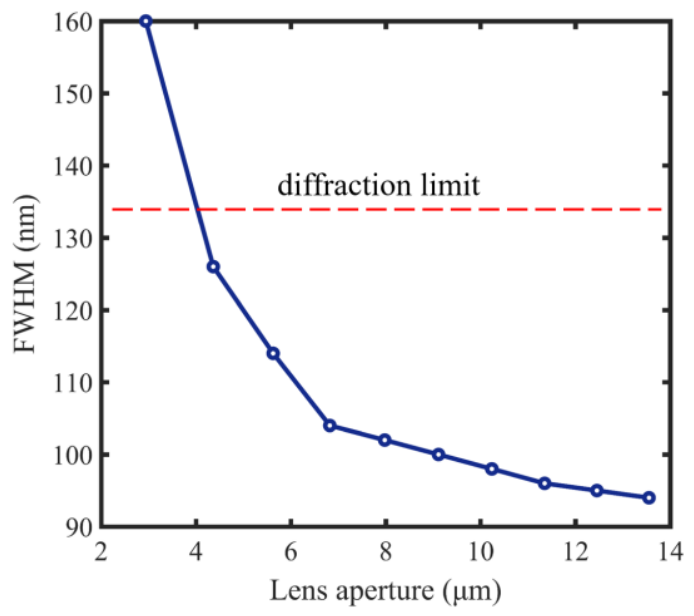

(a)

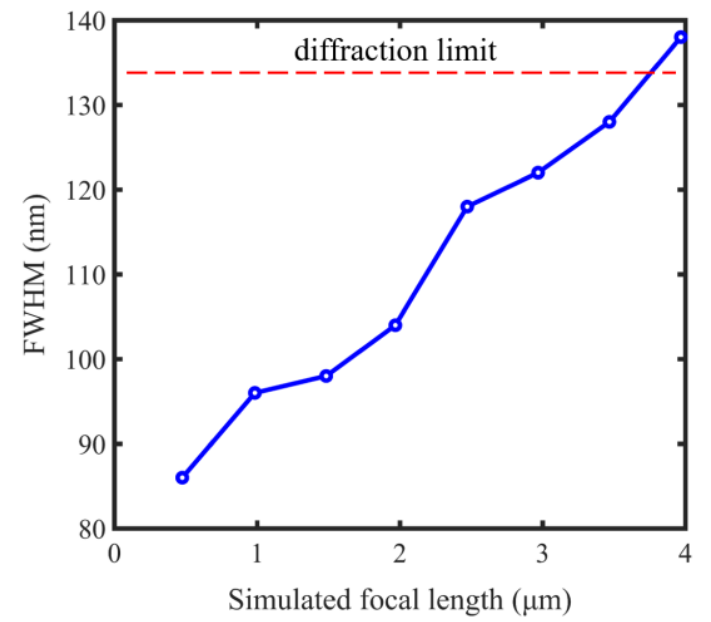

(b)

Figure 5. Effect of lens aperture and focal length on the focal size. (a) Effect of lens aperture on the lens FWHM. The designed focal length of the lens is $1.5 \mu \mathrm{m}$. (b) Effect of focal distance on the lens FWHM.

Focal length also has a great influence on the super-resolution capability of the nanoslit-based 2D lens. Table 2 gives the focusing performance of nanoslit-based lenses with different focal lengths. These lenses were designed with different focal length and nearly identical aperture by using the same design procedure described in Section 2. From Table 2, it can be seen that the FWHM of the lens focus with the focal length of $0.476 \mu \mathrm{m}$ is $86 \mathrm{~nm}(\lambda / 4.71)$, much smaller than the diffraction limit of $134 \mathrm{~nm}$ $(\lambda / 3.03)$, demonstrating an excellent super-resolution focusing ability. However, for the lens with the focal length of $4 \mu \mathrm{m}$, the FWHM is $138 \mathrm{~nm}(\lambda / 2.93)$, larger than the diffraction limit. As a result, the lens cannot break the diffraction limit. The lens with smaller focal length shows better super-resolution focusing ability, as illustrated in Figure 5b. 
Table 2. The derived focusing performance of nanoslit-based lenses with different focal lengths.

\begin{tabular}{cccc}
\hline Designed Focal Length $(\boldsymbol{\mu m})$ & Lens Aperture $(\boldsymbol{\mu m})$ & Simulated Focal Length $(\boldsymbol{\mu m})$ & FWHM $(\mathbf{n m})$ \\
\hline 0.500 & 10.230 & 0.476 & $86(\lambda / 4.71)$ \\
1.000 & 10.564 & 0.984 & $96(\lambda / 4.22)$ \\
1.500 & 10.238 & 1.484 & $98(\lambda / 4.13)$ \\
2.000 & 10.386 & 1.968 & $104(\lambda / 3.89)$ \\
2.500 & 10.482 & 2.472 & $118(\lambda / 3.43)$ \\
3.000 & 10.474 & 2.968 & $122(\lambda / 3.32)$ \\
3.500 & 10.394 & 3.468 & $128(\lambda / 3.12)$ \\
4.000 & 10.230 & 3.968 & $138(\lambda / 2.93)$ \\
\hline
\end{tabular}

\section{Conclusions}

In summary, we explore the effect of various factors on the super-resolution focusing properties of a TE-polarized nanoslit-based 2D lens for guiding practical application. The factor of nanoslit length is firstly considered. The results illustrate that the nanoslit length determines the line focus length of a nanoslit-based 2D lens. The latter is less than the former. Nevertheless, the larger nanoslit length can lead to the smaller deviation of the focus length from it. On the other hand, the focal size of a nanoslit-based 2D lens depends on the two parameters, namely, lens aperture and focal length. When the lens aperture is larger than a certain value, the lens can realize super-resolution focusing. Moreover, the super-resolution capability can be improved as the lens aperture increases. The focal length has a decisive impact on the super-resolution capability of a TE-polarized nanoslit-based 2D lens. A smaller focal length can lead to better super-resolution-focusing performance. When focal length is larger than a certain value, the super-resolution focusing will not be achieved, even though the lens has a large aperture. Our work provides a design guide not only for optimizing a TE-polarized nanoslit-based 2D lens but also for optimizing a TM-polarized nanoslit-based lens [11-17].

Author Contributions: Data curation, S.Z.; Formal analysis, Z.W.; Methodology, Y.Y.; Project administration, W.Y.; Supervision, W.L.; Writing-review \& editing, Y.Z. The manuscript was written through contributions of all authors. All authors have given approval to the final version of this manuscript.

Funding: This research was funded by the National Natural Science Foundation of China (51622509), National Natural Science Foundation of China (51902240), National Natural Science Foundation of China (51975483), the 111 Project (B13044) and General Armament Department Advanced Manufacture of China (41423020111).

Conflicts of Interest: The authors declare no conflict of interest.

\section{References}

1. Abbe, E. Resolution of microscopes. Arch. Mikrosk. Anat. 1873, 9, 413-425. [CrossRef]

2. Zhang, X.; Liu, Z. Superlenses to overcome the diffraction limit. Nat. Mater. 2008, 7, 435-441. [CrossRef] [PubMed]

3. Gramotnev, D.K.; Bozhevolnyi, S.I. Plasmonics beyond the diffraction limit. Nat. Photon. 2010, 4, 83-91. [CrossRef]

4. Barnes, W.L.; Dereux, A.; Ebbesen, T.W. Surface plasmon subwavelength optics. Nature 2003, 424, 824-830. [CrossRef]

5. Fang, N. Sub-Diffraction-Limited Optical Imaging with a Silver Superlens. Science 2005, 308, 534-537. [CrossRef] [PubMed]

6. Gramotnev, D.K.; Bozhevolnyi, S.I. Nanofocusing of electromagnetic radiation. Nat. Photon. 2014, 8, 13-22. [CrossRef]

7. Pan, L.; Park, Y.; Xiong, Y.; Ulin-Avila, E.; Wang, Y.; Zeng, L.; Xiong, S.; Rho, J.; Sun, C.; Bogy, D.B.; et al. Maskless plasmonic lithography at $22 \mathrm{~nm}$ resolution. Sci. Rep. 2011, 1, 175. [CrossRef]

8. Li, Y.H.; Fu, Y.Q.; Minin, O.V.; Minin, I.V. Ultra-sharp nanofocusing of graded index photonic crystal-based lenses perforated with optimized single defect. Opt. Mater. Express 2016, 6, 2628-2636. [CrossRef]

9. Zhu, Y.; Yuan, W.; Sun, H.; Yu, Y. Broadband ultra-deep sub-diffraction-limit optical focusing by metallic graded-index (MGRIN) lenses. Nanomaterials 2017, 7, 221. [CrossRef] 
10. Cao, Y.; Liu, Z.; Minin, O.V.; Minin, I.V. Deep subwavelength-scale light focusing and confinement in nanohole-structured mesoscale dielectric spheres. Nanomaterials 2019, 9, 186. [CrossRef]

11. Shi, H.; Wang, C.; Du, C.; Luo, X.; Dong, X.; Gao, H. Beam manipulating by metallic nano-slits with variant widths. Opt. Express 2005, 13, 6815-6820. [CrossRef] [PubMed]

12. Verslegers, L.; Catrysse, P.B.; Yu, Z.; White, J.S.; Barnard, E.S.; Brongersma, M.L.; Fan, S. Planar lenses based on nanoscale slit arrays in a metallic film. Nano Lett. 2009, 9, 235-238. [CrossRef]

13. Verslegers, L.; Catrysse, P.B.; Yu, Z.; Fan, S. Planar metallic nanoscale slit lenses for angle compensation. Appl. Phys. Lett. 2009, 95, 071112. [CrossRef]

14. Chen, Q.; Cumming, D.R.S. Visible light focusing demonstrated by plasmonic lenses based on nano-slits in an aluminum film. Opt. Express 2010, 18, 14788-14793. [CrossRef] [PubMed]

15. Yu, Y.; Zappe, H. Effect of lens size on the focusing performance of plasmonic lenses and suggestions for the design. Opt. Express 2011, 19, 9434-9444. [CrossRef] [PubMed]

16. Yu, Y.; Zappe, H. Theory and implementation of focal shift plasmonic of lenses. Opt. Lett. 2012, 37, 1592-1594. [CrossRef]

17. Ashari-Bavil, M.; Dong, M.; Li, C.; Feng, S.; Zhu, L. Compact plasmonic lens based on nanoslits in the tree age chart arrangement. Plasmonics 2019, 14, 1161-1167. [CrossRef]

18. Lin, L.; Goh, X.M.; McGuinness, L.P.; Roberts, A. Plasmonic lenses formed by two-dimensional nanometric cross-shaped aperture arrays for fresnel-region focusing. Nano Lett. 2010, 10, 1936-1940. [CrossRef]

19. Ni, X.; Ishii, S.; Kildishev, A.V.; Shalaev, V.M. Ultra-thin, planar, babinet-inverted plasmonic metalenses. Light-Sci. Appl. 2013, 2, 72. [CrossRef]

20. Zhao, L.; Li, Y.; Qi, J.; Xu, J.; Sun, Q. Light focusing by the unique dielectric nano-waveguide array. Opt. Express 2009, 17, 17136-17143. [CrossRef]

21. Arbabi, A.; Horie, Y.; Bagheri, M.; Faraon, A. Dielectric metasurfaces for complete control of phase and polarization with subwavelength spatial resolution and high transmission. Nat. Nanotechnol. 2015, 10, 937-943. [CrossRef] [PubMed]

22. Chong, K.E.; Staude, I.; James, A.; Dominguez, J.; Liu, S.; Campione, S.; Subramania, G.S.; Luk, T.S.; Decker, M.; Neshev, D.N.; et al. Polarization-Independent Silicon Metadevices for Efficient Optical Wavefront Control. Nano Lett. 2015, 15, 5369-5374. [CrossRef] [PubMed]

23. Lin, D.; Fan, P.; Hasman, E.; Brongersma, M.L. Dielectric gradient metasurface optical elements. Science 2014, 345, 298-302. [CrossRef] [PubMed]

24. Aieta, F.; Kats, M.A.; Genevet, P.; Capasso, F. Multiwavelength achromatic metasurfaces by dispersive phase compensation. Science 2015, 347, 1342-1345. [CrossRef]

25. Arbabi, A.; Horie, Y.; Ball, A.J.; Bagheri, M.; Faraon, A. Subwavelength-thick lenses with high numerical apertures and large efficiency based on high-contrast transmitarrays. Nat. Commun. 2015, 6, 7069. [CrossRef]

26. Khorasaninejad, M.; Chen, W.T.; Devlin, R.C.; Oh, J.; Zhu, A.Y.; Capasso, F. Metalenses at visible wavelengths: Diffraction-limited focusing and subwavelength resolution imaging. Science 2016, 352, 1190-1194. [CrossRef]

27. Chen, W.T.; Zhu, A.Y.; Sanjeev, V.; Khorasaninejad, M.; Shi, Z.; Lee, E.; Capasso, F. A broadband achromatic metalens for focusing and imaging in the visible. Nat. Nanotechnol. 2018, 13, 220-226. [CrossRef]

28. Mote, R.G.; Minin, O.V.; Minin, I.V. Focusing behavior of 2-dimensional plasmonic conical zone plate. Opt. Quant. Electron. 2017, 49, 271. [CrossRef]

29. Zhu, Y.; Yuan, W.; Li, W.; Sun, H.; Qi, K.; Yu, Y. TE-polarized design for metallic slit lenses: a way to deep-subwavelength focusing over a broad wavelength range. Opt. Lett. 2018, 43, 206-209. [CrossRef]

30. Johnson, P.B.; Christy, R.W. Optical constants of the noble metals. Phys. Rev. B 1972, 6, 4370-4379. [CrossRef]

(C) 2019 by the authors. Licensee MDPI, Basel, Switzerland. This article is an open access article distributed under the terms and conditions of the Creative Commons Attribution (CC BY) license (http://creativecommons.org/licenses/by/4.0/). 